\title{
Tratamento farmacológico para endometriose
}

\section{Pharmacological treatment for endometriosis \\ Tratamiento farmacológico para la endometriosis}

Camila Maria do Nascimento Santos

ORCID: https://orcid.org/0000-0003-3796-0710

Associação de Ensino Superior do Piauí, Brasil

E-mail: camilamarisantos9892@gmail.com

Luiz Eduardo Macêdo do Monte

ORCID: https://orcid.org/0000-0002-5264-0371

Associação de Ensino Superior do Piauí, Brasil

E-mail: luizeduardomacedodomonte@gmail.com

José Chagas Pinheiro Neto

ORCID: https://orcid.org/0000-0002-6626-6122 Universidade Federal do Piaú, Brasil

E-mail: jose.cpneto@hotmail.com

Higo José Neri da Silva

ORCID: https://orcid.org/0000-0003-2089-4147 Universidade Federal do Piauí, Brasil E-mail: higoneri@gmail.com

Antonia Filha Moreira de Melo

ORCID: https://orcid.org/0000-0002-0690-4380

Centro Universitário UNINOVAFAPI, Brasil E-mail: afmdemello@gmail.com

Sérgio Henrique Assunção Lacerda Borges ORCID: https://orcid.org/0000-0002-8553-7223

Centro Universitário UNINOVAFAPI, Brasil E-mail: seergiol@hotmail.com

Alisson Camilo Martins Sousa

ORCID: https://orcid.org/0000-0003-4613-5114 Graduando em Farmácia, Brasil

E-mail: alissoncamilo1986@hotmail.com

Juciara Carvalho de Oliveira

ORCID: https://orcid.org/0000-0002-2010-2587

Laboratório Central do Piauí, Brasil E-mail: kelly-rego@bol.com.br

Fabiana de Souza Santana

ORCID: https://orcid.org/0000-0002-4395-773X

Faculdade das Américas, Brasil

E-mail: fabianasantana6@ hotmail.com

Anna Letícia de Sousa Marinho

ORCID: https://orcid.org/0000-0002-6021-5981

Centro Universitário UNINOVAFAPI, Brasil

E-mail: lelesmarinho99@gmail.com

Márcia Araújo Gualberto

ORCID: https://orcid.org/0000-0001-5102-6241 Hospital São Marcos, Brasil

E-mail: marciagualberto@hotmail.com

Ian Jhemes Oliveira Sousa

ORCID: https://orcid.org/0000-0003-1930-9828 Universidade Federal do Piauí, Brasil E-mail: ianjhemes@gmail.com

Rodrigo Lopes Gomes Gonçalves ORCID: https://orcid.org/0000-0001-6482-4998 Universidade Federal do Piauí, Brasil

E-mail: profrodrigolgoncalves@ gmail.com

Kelly Maria Rêgo da Silva

ORCID: https://orcid.org/0000-0003-3070-4060

Laboratório Central do Piauí, Brasil

E-mail: kellyma.aria@gmail.com

Iluska Martins Pinheiro

ORCID: https://orcid.org/0000-0002-8160-9787

Laboratório Central do Piauí, Brasil

E-mail: iluskasmartins@gmail.com 


\begin{abstract}
Resumo
A endometriose é uma doença ginecológica que se caracteriza pela presença de endométrio como tecido-epitélio e estroma que se desenvolve fora da cavidade uterina. É uma doença crónica, estrogênio-dependente, é associada com dor pélvica, dismenorreia, dispareunia e infertilidade. Dado o grande impacto desta doença na qualidade de vida das mulheres, tem-se verificado um interesse crescente na descoberta de novos fármacos mais eficazes, tolerabilidade e segurança. Para isso, realizou-se uma revisão da literatura a partir de artigos publicados entre os anos de 2008 a 2018 disponíveis a respeito dos Tratamentos farmacológicos para endometriose, nas bases de dados SCIENCE DIRECT e PUBMED. Encontrou-se um total de 223 artigos publicados nos últimos 10 anos, em "português e inglês". Após avaliação dos artigos, foram selecionados 15 artigos para o presente estudo, que cumpriram com todos os critérios de elegibilidade proposto na metodologia. Concluiu-se que podemos contar com vários tipos de tratamentos para endometriose, mas que a maioria tem como objetivo tratar os sintomas associados à endometriose. Atualmente o tratamento específico que podemos contar é o Dienogeste. Além disso, existem muitos estudos em busca de novas drogas e tratamentos promissores para essa patologia. Assim, fazem-se necessárias mais pesquisas a respeito do tema e que o farmacêutico tem papel fundamental para a adesão ao tratamento do paciente.
\end{abstract}

Palavras-chave: Tratamento farmacológico; Endometriose; Terapia combinada.

\begin{abstract}
Endometriosis is a gynecological disease characterized by the presence of endometrium as tissue-epithelium and stroma that develops outside the uterine cavity. It is a chronic, estrogen-dependent disease, is associated with pelvic pain, dysmenorrhea, dyspareunia and infertility. Given the great impact of this disease on the quality of life of women, there has been a growing interest in the discovery of new drugs that are more effective, tolerability and safety. For this, a literature review was carried out from articles published between 2008 and 2018 available regarding pharmacological treatments for endometriosis in the SCIENCE DIRECT and PUBMED databases. A total of 223 articles published in the last 10 years were found in "Portuguese and English". After evaluating the articles, 15 articles were selected for the present study, which complied with all the eligibility criteria proposed in the methodology. It was concluded that we can count on several types of treatments for endometriosis, but most have the objective of treating the symptoms associated with endometriosis. Currently, the specific treatment we can count on is dienogeste. In addition, there are many studies in search of new drugs and promising treatments for this pathology. Thus, more research is needed regarding the theme and that the pharmacist plays a fundamental role in the adherence to the patient's treatment.
\end{abstract}

Keywords: Pharmacological treatment; Endometriosis; Combined therapy.

\title{
Resumen
}

La endometriosis es una enfermedad ginecológica que se caracteriza por la presencia de endometrio como tejidoepitelio y estroma que se desarrolla fuera de la cavidad uterina. Es una enfermedad crónica dependiente de estrógenos, asociada a dolor pélvico, dismenorrea, dispareunia e infertilidad. Dado el gran impacto de esta enfermedad en la calidad de vida de las mujeres, ha habido un interés creciente en el descubrimiento de fármacos nuevos, más eficaces, de tolerabilidad y seguridad. Para ello, se realizó una revisión de la literatura a partir de los artículos publicados entre los años 2008 a 2018 disponibles sobre los tratamientos farmacológicos para la endometriosis, en las bases de datos SCIENCE DIRECT y PUBMED. Se encontraron un total de 223 artículos publicados en los últimos 10 años en "portugués e inglés". Luego de evaluar los artículos, se seleccionaron 15 artículos para el presente estudio, los cuales cumplieron con todos los criterios de elegibilidad propuestos en la metodología. Se concluyó que podemos contar con varios tipos de tratamientos para la endometriosis, pero que la mayoría de ellos tienen como objetivo tratar los síntomas asociados a la endometriosis. Actualmente el tratamiento específico con el que podemos contar es el Dienogeste. Además, son muchos los estudios que buscan nuevos fármacos y tratamientos prometedores para esta patología. Por tanto, se necesita más investigación sobre el tema y que el farmacéutico tiene un papel fundamental en la adherencia al tratamiento del paciente.

Palabras clave: Tratamiento farmacológico; Endometriosis; Terapia combinada.

\section{Introdução}

No processo fértil da mulher, todos os meses o corpo se prepara para o momento em que vai ocorrer uma possível fecundação (o ciclo menstrual). Os ovários produzem hormônios que estimulam as células da mucosa do útero (endométrio) a se multiplicarem e estarem preparadas para receberem um óvulo fertilizado. A mucosa aumenta de tamanho e fica mais espessa. Quando não ocorre a gravidez, o endométrio que aumentou descama e é expelido na menstruação (Febrasgo, 2017).

Ao contrário das células normalmente encontradas dentro do útero que são liberadas durante a menstruação, células endometriais podem crescer fora do útero, surgindo à endometriose que é uma enfermidade ginecológica, de difícil diagnóstico, com falta de tratamento clínico efetivo. É uma patologia que afeta mulheres em idade reprodutiva, ou seja, entre a 
menarca e a menopausa. Causa lesões em diversos locais próximos a região. Os órgãos mais acometidos pelas lesões estão localizados na pélvis, ligamentos, ligamento sacro uterino e saco de Douglas (Durón \& Bolanõs, 2018).

Existem várias teorias que explicam a patogênese da endometriose, como a teoria metaplásica celômica, teoria da indução, teoria dos restos embrionários, teoria da extensão direta, teoria da metástase linfática e vascular, teoria da transplantação, implantação do tecido endometrial ou menstruação retrógrada. Todas apontam para a multicausalidade associado a fatores genéticos, anormalidades imunológicas e disfunção endometrial (Brasil, 2002).

No entanto, a teoria mais aceita é a teoria da implantação, que foi descrita por Sampson em 1925, por se tratar de uma explicação mais coerente, onde através de menstruação retrógrada que ocorre fisiologicamente, ocorre que o tecido endometrial ganha acesso às estruturas pélvicas através das tubas uterinas implantando-se na superfície peritoneal, estabelecendo fluxo sanguíneo e gerando resposta inflamatória (Santos et al., 2012).

A endometriose é uma doença enigmática, atualmente é classificada de forma que procura identificar a localização das lesões, o grau de comprometimento dos órgãos e a severidade da doença. Numerosos sistemas para classificação ou estadiamento da endometriose foram propostos até hoje. O mais comumente empregado é a classificação revisada da American Society of Reproductive Medicine (Khazali, 2016).

Para o tratamento da endometriose podemos contar com um leque de opções, sendo necessário adotar um plano de gestão em longo prazo, com o objetivo de maximizar o uso de tratamento médico e evitar procedimentos cirúrgicos repetidos, e por seus mecanismos envolvidos serem numerosos e sua compreensão está em constante evolução. A farmacoterapia de primeira linha age bloqueando a função ovariana, criando um ambiente hipoestrogênico. Este tipo de tratamento clínico envolve alterações hormonais com objetivo de produzir um estado de pseudogravidez, pseudomenopausa ou anovulação crônica. $\mathrm{O}$ estado de hipoestrogenismo provocado por estas drogas induz a atrofia do endométrio ectópico além de permitir o controle dos sintomas dolorosos possivelmente por diminuição da produção de prostaglandinas e citocinas com menor estimulação das fibras nervosas. Por isso reforça-se a necessidade de individualização em cada tratamento (Clemenza, 2018).

Deste modo, o estudo objetiva-se através desta revisão de literatura, analisar os aspectos farmacoterapêuticos da endometriose, apresentando os principais tratamentos farmacológicos disponíveis no mercado, além de descrever a importância da atenção farmacêutica na adesão ao tratamento da doença.

\section{Metodologia}

\subsection{Método da pesquisa}

Trata-se de uma revisão bibliográfica, descritiva, de caráter quali-quantitativo.

\subsection{Coleta de dados}

A pesquisa bibliográfica consistiu na análise de artigos científicos nos bancos de dados publicados entre 2008 a 2018, disponíveis online na íntegra a respeito da farmacologia usada no tratamento da endometriose seguindo metodologia adaptada de Pereira et al., (2018). Os artigos foram coletados nos seguintes bancos de dados: SCIENCE DIRECT e PUBMED. Foram utilizados os descritores em combinação: "Tratamento Farmacológico", "Endometriose", "Terapia Combinada" em português e inglês, utilizando o conectivo "and" para o cruzamento entre as palavras. Como critérios de inclusão, foram utilizados artigos que explanaram sobre o objetivo definido, artigos completos publicados nos anos delimitados em português e inglês. Como critérios de exclusão não foram utilizados artigos que não abordavam sobre os tratamentos farmacológicos da endometriose, artigos de revisão assim como aqueles em duplicidade, e que não se encontram disponíveis de forma completa na íntegra. 


\subsection{Análises dos dados}

A triagem dos artigos deu-se através da leitura criteriosa e integral do material bibliográfico por dois pesquisadores. Realizou-se uma leitura exploratória dos títulos e resumos, sendo selecionados e analisados integralmente apenas aqueles artigos relacionados ao propósito do estudo. Após a análise, os resultados foram organizados utilizando-se os programas Microsoft Word e Excel 2010.

\section{Resultados e Discussão}

Realizando-se uma busca nas bases de dados SCIENCE DIRECT e PUBMED foram encontrados no total 223 artigos que englobam o tema Tratamento Farmacológico, Endometriose e Terapia Combinada, publicados nos últimos 10 anos, em português e inglês. A distribuição de artigos por referida base de dados é apresentada na Tabela 1.

Tabela 1: Artigos disponíveis publicados nas bases de dados no período de 2008 a 2018, aplicando os descritores: "Endometriose", "Tratamento Farmacológico", "Terapia Combinada", utilizando o conectivo "and" para o cruzamento das palavras nas pesquisas.

\begin{tabular}{|c|c|c|}
\hline BASES DE DADOS & SCIENCE DIRECT & PUBMIDD \\
\hline Endometriose and tratamento farmacológico & 71 artigos & 130 artigos \\
\hline Endometriose and terapia combinada & 10 artigos & 12 artigos \\
\hline Total & 81 artigos & 142 artigos \\
\hline Selecionados & 6 artigos & 9 artigos \\
\hline
\end{tabular}

Fonte: Dados da Pesquisa (2018).

Apesar do elevado número de publicações, observa-se que, em sua maioria, os artigos estavam relacionados a qualidade de e vida de mulheres com endometriose, ao câncer e novas terapias em estudos iniciais. Além disso, muitos artigos, apesar de citarem a endometriose, apresentaram uma diferente temática central. Dessa forma, tais fatores levaram à exclusão de diversos artigos do presente estudo.

A Tabela 2 mostra os estudos selecionados que estão sumarizados considerando informações como, Título, autor (es), ano, periódico de publicação, objetivos. Os artigos analisados foram organizados de forma a favorecer a melhor leitura dos resultados.

Tabela 2: Caracterização de artigos selecionados quanto a suas informações individuais.

\begin{tabular}{|c|c|c|c|}
\hline \multicolumn{1}{|c|}{ Título } & Autor / Ano & \multicolumn{2}{c|}{ Objetivo do Estudo } \\
\hline $\begin{array}{c}\text { Ensaio clínico randomizado duplo } \\
\text { cego com resveratrol no tratamento } \\
\text { da dor por endometriose. }\end{array}$ & SILVA, D. (2017) & $\begin{array}{c}\text { Em comparação com placebo, o resveratrol } \\
\text { (40mg/dia) reduz níveis de dor após 42 dias } \\
\text { de uso em mulheres com endometriose } \\
\text { usando pílula anticoncepcional monofásica } \\
\text { (PAM) }\end{array}$ & UFRG \\
\hline $\begin{array}{c}\text { Avaliação da qualidade de vida de } \\
\text { pacientes portadoras de endometriose } \\
\text { após inserção do Sistema Intra- } \\
\begin{array}{c}\text { Uterino Liberador de Levonorgestrel } \\
\text { (SIU-LNg). }\end{array}\end{array}$ & $\begin{array}{c}\text { FIGUEIREDO, J. \& } \\
\text { NASCIMENTO, R. }\end{array}$ & $\begin{array}{c}\text { Avaliar a qualidade de vida das mulheres } \\
\text { com endometriose através da aplicação de } \\
\text { um questionário estruturado, antes e após a } \\
\text { inserção do SIU-LNg durante seis meses de } \\
\text { seguimento. }\end{array}$ & $\begin{array}{c}\text { Associação } \\
\text { Catanense de } \\
\text { Medicina }\end{array}$ \\
\hline $\begin{array}{c}\text { Induction of a local pseudo- } \\
\text { pregnancy for the treatment of } \\
\text { endometriosis. }\end{array}$ & YUAN, P. et al., (2010) & $\begin{array}{c}\text { Induzir uma pseudo-gravidez local via } \\
\text { microesferas carregadas de progestogênio } \\
\text { nas lesões de endometriose }\end{array}$ & Medical Hypotheses \\
\hline
\end{tabular}




\begin{tabular}{|c|c|c|c|}
\hline $\begin{array}{l}\text { Gonadotropin-releasing hormone } \\
\text { analogs: understanding advantages } \\
\text { and limitations. }\end{array}$ & $\begin{array}{c}\text { KUMAR, P. \& } \\
\text { SHARMA, A. (2014) }\end{array}$ & $\begin{array}{c}\text { Apresentar uma visão geral do uso de } \\
\text { análogos de GnRH, que são potentes } \\
\text { agentes terapêuticos e consideravelmente } \\
\text { úteis em uma variedade de indicações } \\
\text { clínicas. }\end{array}$ & $\begin{array}{l}\text { Journal of Human } \\
\text { Reproductive } \\
\text { Sciences }\end{array}$ \\
\hline $\begin{array}{l}\text { Selective progesterone receptor } \\
\text { modulators and progesterone } \\
\text { antagonists: mechanisms of action } \\
\text { and clinical applications. }\end{array}$ & $\begin{array}{l}\text { CHABBERT-BUFFET, } \\
\text { N. et al., (2008) }\end{array}$ & $\begin{array}{l}\text { Destacar o mecanismo de ação destes } \\
\text { compostos com especial ênfase em seus } \\
\text { efeitos no útero. }\end{array}$ & $\begin{array}{c}\text { Human } \\
\text { Reproduction }\end{array}$ \\
\hline $\begin{array}{l}\text { Aromatase inhibition for refractory } \\
\text { endometriosis-related chronic pelvic } \\
\text { pain. }\end{array}$ & ABUSHAHIN, F. (2011) & $\begin{array}{l}\text { Avaliar o uso de um inibidor de aromatase } \\
\text { para o tratamento da dor pélvica crônica } \\
\text { relacionada à endometriose }\end{array}$ & $\begin{array}{l}\text { Fertility and } \\
\text { Sterility }\end{array}$ \\
\hline $\begin{array}{l}\text { Dual suppression of estrogenic and } \\
\text { inflammatory activities for targeting } \\
\text { of endometriosis. }\end{array}$ & ZHAO, Y. et al., (2015) & $\begin{array}{l}\text { Avaliar a eficácia de dois ligantes ER na } \\
\text { prevenção da lesão endometriose } \\
\text { estabelecimento e na indução de regressão } \\
\text { de lesão em modelo de camundongo, e } \\
\text { examinar os mecanismos subjacentes aos } \\
\text { seus efeitos antiproliferativos e anti- } \\
\text { inflamatórios atividades. }\end{array}$ & $\begin{array}{l}\text { Science } \\
\text { Translational } \\
\text { Medicine }\end{array}$ \\
\hline $\begin{array}{c}\text { Biological characterization of a novel, } \\
\text { orally active small molecule } \\
\text { gonadotropin-releasing hormone } \\
\text { (GnRH) antagonist using castrated } \\
\text { and intact rats. }\end{array}$ & $\begin{array}{l}\text { ANDERES, K. L. et al., } \\
\text { (2008) }\end{array}$ & $\begin{array}{c}\text { Caracterização biológica de um novo } \\
\text { antagonista do hormônio liberador de } \\
\text { gonadotrofina }(\mathrm{GnRH})\end{array}$ & $\begin{array}{c}\text { Journal } \\
\text { of Pharmacology }\end{array}$ \\
\hline $\begin{array}{l}\text { Satisfação e padrões de sangramento } \\
\text { das usuárias do sistema intrauterino } \\
\text { liberador de levonorgestrel como } \\
\text { contraceptivo e comparação com o } \\
\text { implante contraceptivo liberador de } \\
\text { etonogestrel em mulheres com dor } \\
\text { pélvica associada à endometriose. }\end{array}$ & $\begin{array}{l}\text { TAVARES, N. M. C. } \\
\text { (2018) }\end{array}$ & $\begin{array}{l}\text { Avaliar a relação entre a satisfação de } \\
\text { usuárias de SIU-LNG e o padrão de } \\
\text { sangramento relatado em dois momentos } \\
\text { durante o seguimento e comparar a eficácia } \\
\text { terapêutica do SIU-LNG e implante } \\
\text { liberador de etonogestrel (ENG) no controle } \\
\text { da dor pélvica, dismenorreia, padrão de } \\
\text { sangramento. }\end{array}$ & UNICAMP \\
\hline $\begin{array}{l}\text { Dopamine agonist administration } \\
\text { causes a reduction in endometrial } \\
\text { implants through modulation of } \\
\text { angiogenesis in experimentally } \\
\text { induced endometriosis. }\end{array}$ & $\begin{array}{l}\text { NOVELLA-MAESTRE, } \\
\text { E. et al., (2009) }\end{array}$ & $\begin{array}{l}\text { Avaliar as propriedades anti-angiogênicas } \\
\text { de } \mathrm{Cb} 2 \text { no crescimento de lesões de } \\
\text { endometriose estabelecidas e investigar os } \\
\text { mecanismos moleculares pelos quais } \mathrm{Cb} 2 \\
\text { exerce o efeito anti-angiogênico. }\end{array}$ & $\begin{array}{c}\text { Human } \\
\text { Reproduction }\end{array}$ \\
\hline $\begin{array}{l}\text { Short-term histopathological effects } \\
\text { of dienogest therapy on ovarian } \\
\text { endometriomas: in vivo, non- } \\
\text { randomized, controlled trial. }\end{array}$ & $\begin{array}{l}\text { MABROUK, M. et al., } \\
\text { (2018) }\end{array}$ & $\begin{array}{l}\text { Avaliar os principais efeitos } \\
\text { histopatológicos da terapia com dienogest } \\
\text { em curto prazo em pacientes com } \\
\text { endometriomas ovarianos agendados para } \\
\text { cirurgia. }\end{array}$ & $\begin{array}{l}\text { Gynecol } \\
\text { Endocrinol. }\end{array}$ \\
\hline $\begin{array}{l}\text { Current Strategies for Endometriosis } \\
\text { Management. }\end{array}$ & $\begin{array}{l}\text { KODAMAN, P. H. } \\
\text { (2015) }\end{array}$ & $\begin{array}{c}\text { Definir os tratamentos atuais para } \\
\text { endometriose }\end{array}$ & $\begin{array}{l}\text { Obstetrics and } \\
\text { Gynecology }\end{array}$ \\
\hline $\begin{array}{l}\text { Treatment of Endometriosis- } \\
\text { Associated Pain with Elagolix, an } \\
\text { Oral GnRH Antagonist. }\end{array}$ & $\begin{array}{l}\text { TAYLOR, H. S. et al., } \\
\text { (2017) }\end{array}$ & $\begin{array}{l}\text { Realizar dois ensaios clínicos de fase } 3 \text { de } 6 \\
\text { meses, duplo-cegos, para avaliar os efeitos } \\
\text { de duas doses de elagolix - } 150 \text { mg e } 200 \\
\text { mg em comparação com o placebo em } \\
\text { mulheres com diagnosticado cirurgicamente } \\
\text { a endometriose e moderada ou grave. }\end{array}$ & $\begin{array}{l}\text { The New England } \\
\text { journal of medicine }\end{array}$ \\
\hline $\begin{array}{l}\text { From pathogenesis to clinical } \\
\text { practice: Emerging medical } \\
\text { treatments for endometriosis. }\end{array}$ & $\begin{array}{l}\text { CLEMENZA, S. et al., } \\
(2018)\end{array}$ & $\begin{array}{l}\text { Novas alternativas terapêuticas hormonais e } \\
\text { não hormonais aos tratamentos padrão. }\end{array}$ & $\begin{array}{l}\text { Obstetrics and } \\
\text { Gynaecology }\end{array}$ \\
\hline $\begin{array}{l}\text { Aspectos diagnósticos e terapêuticos } \\
\text { da endometriose. }\end{array}$ & AMARAL, P. P. (2017) & $\begin{array}{l}\text { Apresentar os principais aspectos } \\
\text { diagnósticos e terapêuticos da } \\
\text { endometriose. }\end{array}$ & Faema \\
\hline
\end{tabular}

Fonte: Dados da Pesquisa (2018).

Figueiredo e Nascimento, (2008) afirmam em seu estudo que o tratamento médico da endometriose é predominantemente paliativo para os sintomas mais comuns como dismenorreia, dispareunia, dor pélvica não cíclica e/ou 
menorragia. O uso da terapia medicamentosa para endometriose é baseado no fato de ser responsiva aos hormônios, ou seja, aos estrogênios, proliferando o endométrio.

Os tratamentos farmacológicos para endometriose agem bloqueando a função ovariana, suprimindo a menstruação e induzindo atrofia endometrial. Sua principal limitação é o efeito contraceptivo para mulheres que buscam uma gravidez. Tratamentos médicos com agonistas do hormônio liberador de gonadotrofinas (GnRH), inibidores de aromatases (AIs), moduladores seletivos do receptor de progesterona (SPRMs) e drogas antiangiogênicas são muito promissoras (Clemenza et al., 2018).

Para Silva (2017) o tratamento farmacológico da endometriose, em especial as estratégias relacionadas ao manejo da dor, vem apresentando ao longo das últimas décadas várias modificações, porém sempre focando em regimes hormonais, que quando cessados permitem o retorno de sintomas álgicos.

\subsection{Tratamentos hormonais}

A terapia hormonal é a base no tratamento médico de endometriose. Os objetivos do tratamento incluem o alívio da dor e prevenção de recorrência. Esses agentes suprimem síntese de estrogênio, reduzir o sangramento, bem como incluir atrofia dos implantes endometrióticos, criando um "pseudo gravidez" ou "pseudo-menopausa". Embora todos agentes sejam eficazes, os mesmos diferem em seu mecanismo de ação, via de administração, duração da terapia e seus efeitos (YUAN et. al., 2010).

\subsection{Agonistas do hormônio liberador de gonadotrofinas (GnRH)}

Agonistas dos receptores do hormônio liberador de gonadotropina $(\mathrm{GnRH})$ tais como cetrorelix, elagolix, ganirelix, abarelix e degarelix são amplamente utilizados (Anderes et al., 2008). Os antagonistas de GnRH regula liberação de hormônio folículo-estimulante (FSH) e luteinizante hormônio (LH), bloqueando o receptor de GnRH nas células hipofisárias resultando na supressão da ovulação (Kumar \& Sharma, 2014).

Em um estudo realizado por Taylor et al., (2017), que avaliaram os efeitos de duas doses de elagolix - $150 \mathrm{mg}$ uma vez ao dia (grupo dose) e $200 \mathrm{mg}$ duas vezes ao dia (grupo de dose máxima), em mulheres diagnosticadas com endometriose, observaram que tanto as doses mais altas quanto as doses mais baixas de elagolix foram efetivas na melhora da dismenorréia e da dor pélvica não menstrual durante um período de 6 meses em mulheres com dor associada à endometriose. As duas doses de elagolix foram associadas a efeitos adversos hipoestrogênicos.

\subsection{Inibidores de aromatases (AIs)}

A enzima aromatase é a chave principal na síntese de estrogênio. As AIs suprimem a atividade da enzima aromatase resultando na inibição da conversão de androstenediona e testosterona a estradiol e estrogênio. A atividade da aromatase é aumentada no tecido endometrial extra-uterino que resulta em maior conversão de testosterona em estrogênio. Vários estudos têm investigado que a segurança e eficácia da (AIs) como letrozol e anastrozol como uma terapia única e em conjunto com outras classes de medicamentos, como contraceptivos orais (OCPs), agonistas de GnRH, progestinas e danazol para o tratamento de endometriose (Abushahin, 2011; Zhao et al., 2015).

\subsection{Moduladores seletivos do receptor de progesterona (SPRMs)}

Os SPRMs são agonistas puros, antagonistas e também mistos, com atividade agonista / antagonista. SPRMs melhora os sintomas de endometriose suprimindo a ovulação, exibindo efeito antiproliferativos no endométrio e inibindo o sangramento do mesmo (Chabbert-Buffet et al., 2008).

Até o momento, os progestágenos são recomendados como tratamento médico de primeira linha para endometriose 
ovariana sintomática (Mabrouk et al., 2018). Uma droga bastante utilizada na prática clínica para o tratamento da endometriose é o Dienogest. Segundo Kodaman (2015), vários estudos mostram que o dienogest melhora a dor pélvica no contexto da endometriose, com efeitos benéficos persistentes por 6 meses após a sua interrupção. Além disso, dienogest não possui os efeitos colaterais androgênicos comuns.

\subsection{Tratamentos promissores (antiangiogênicos)}

O líquido peritoneal de mulheres com endometriose consiste em fatores pró-angiogênicos, como fator de crescimento endotelial vascular (VEGF), interleucina (IL) -8 e fator de crescimento da placenta. $\mathrm{O}$ fator de crescimento endotelial vascular é uma proteína sinalizadora produzida pelas células que estimulam o processo de angiogênese e vasculogênese e desempenha um papel vital na endometriose. A quantidade de (VEGF) presente nos fluidos peritoneais correlaciona-se positivamente com a gravidade da doença (Novella-Maestre et al., 2009).

Diversos tratamentos para a endometriose têm sido propostos, incluindo tratamentos cirúrgicos com exérese das lesões e restauração da anatomia pélvica, e tratamentos medicamentosos, drogas angiogênicas como estatinas, moduladores da metaloproteinases de matriz (MMP), acetato de medroxiprogesterona, (Novella-Maestre et al., 2009).

A maioria dos estudos realizados é experimental e provou ser eficaz em estudos com animais. Assim, mais estudos são necessários para apoiar sua introdução na prática clínica de rotina.

\subsection{Papel do farmacêutico no tratamento da endometriose}

Segundo Amaral (2017), o papel do farmacêutico é orientar as pacientes, para que procurem um ginecologista para diagnóstico correto e caso diagnosticado, esclarecer as dúvidas sobre o tratamento e os medicamentos, desde cumprir a posologia adequada, o tempo determinado e os intervalos apropriados, interações medicamentosas, efeitos colaterais, a importância de seguir o tratamento de modo correto e reforçar informações para desfazer mitos e possíveis medos. Também incentivar a comunicação da paciente com os outros membros da equipe visando um acompanhamento multiprofissional.

\section{Conclusão}

Poucas publicações evidenciam sobre os tratamentos para endometriose. Apesar de uma considerável variedade de tratamentos farmacológicos, não existem muitos medicamentos realmente específicos para essa patologia. Existem potenciais fármacos como os antiangiogênicos, que ainda estão em fases de estudos, onde futuramente podem ser utilizados no tratamento da endometriose. O Dienogest é atualmente o fármaco mais utilizado na pratica clínica por ser mais especifico, e não apresentar efeitos colaterais comuns em outros fármacos.

O farmacêutico tem papel fundamental para a adesão ao tratamento do paciente, esclarecendo dúvidas recorrentes sobre a terapia, informando efeitos colaterais comuns e enfatizando a importância de seguir o tratamento de modo correto, respeitando os horários e tempo determinados para o tratamento.

\section{Referências}

Abushahin, F. (2011). Aromatase inhibition for refractory endometriosis-related chronic pelvic pain. Fertil. Steril, 96, 939-942.

Amaral, P. P. (2017). Aspectos diagnósticos e terapêuticos da endometriose. FAEMA. Ariquemes-RO.

Anderes, K. L., et al. (2008). Biological characterization of a novel, orally active small molecule gonadotropin-releasing hormone (GnRH) antagonist using castrated and intact rats. J. Pharmacol, 305, 688-695.

Brasil. Ministério da Saúde. (2002). Política Nacional de Promoção da Saúde (Documento para discussão). Secretaria de Políticas Públicas 
Research, Society and Development, v. 10, n. 7, e52810716104, 2021

(CC BY 4.0) | ISSN 2525-3409 | DOI: http://dx.doi.org/10.33448/rsd-v10i7.16104

Chabbert-Buffet, N., et al. (2008). Selective progesterone receptor modulators and progesterone antagonists: mechanisms of action and clinical applications, Hum. Reprod. 11, 293-307.

Clemenza, S., et al., (2018). From pathogenesis to clinical practice: Emerging medical treatments for endometriosis, Best Practice \& Research Clinical. Obstetrics and Gynaecology.

Durón, G. R., \& Bolaños, M. P. (2018). Endometriosis.

Febrasgo - (2017). Federação Brasileira das Associações de Ginecologia e Obstetrícia

Figueiredo, J., Nascimento, R. Avaliação da qualidade de vida de pacientes portadoras de endometriose após inserção do Sistema Intra-Uterino Liberador de Levonorgestrel (SIU-LNg). ACM, 37, 4

Khazali, S. (2016). Endometriosis Classification-The Quest for the Holy Grail? J Reprod Infertil; 17.

Kodaman, P. H. (2015). Current Strategies for Endometriosis Management. Obstetrics and Gynecology Clinics of North America, $42,87-101$.

Kumar, P., \& Sharma, A. (2014). Gonadotropin-releasing hormone analogs: understanding advantages and limitations, J. Hum. Reprod, 7, 170-174.

Mabrouk, M., et al. (2018). Short-term histopathological effects of dienogest therapy on ovarian endometriomas: in vivo, nonrandomized, controlled trial. Gynecol Endocrinol, v. 34(5); 399-403.

Novella-Maestre, E., et al., (2009). Dopamine agonist administration causes a reduction in endometrial implants through modulation of angiogenesis in experimentally induced endometriosis. Hum. Reprod, 24, 1025-1035.

Pereira, A. S., et al. (2018). Metodologia da pesquisa científica. UFSM.

Santos, D. B., et al. (2012). Uma abordagem integrada da endometriose. UFRB

Silva, D. M. (2017). Ensaio clínico randomizado duplo cego com resveratrol no tratamento da dor por endometriose. Universidade Federal do Rio Grande do Sul.

Tavares, N. M. C. (2018). Satisfação e padrões de sangramento das usuárias do sistema intrauterino liberador de levonorgestrel como contraceptivo e comparação com o implante contraceptivo liberador de etonogestrel em mulheres com dor pélvica associada à endometriose. 2018.1 recurso online (98 p.). Tese (doutorado) - Universidade Estadual de Campinas, Faculdade de Ciências Médicas, <http://www.repositorio.unicamp.br/handle/REPOSIP/331112>

Taylor, H. S., et al. (2017). Treatment of Endometriosis-Associated Pain with Elagolix, an Oral GnRH Antagonist. The New England journal of medicine, 377, $28-40$.

Yuan, P., et. al., (2010). Induction of a local pseudo-pregnancy for the treatment of endometriosis. Medical Hypotheses, 74; 56-58

Zhao, Y., et al., (2015). Dual suppression of estrogenic and inflammatory activities for targeting of endometriosis, Transl. Med, 7; 271. 